\title{
Risk Factors for Puncture Site Original Complications Using the Angio-Seal Closure Device in Endovascular Therapy: A Single-center Analysis
}

Takahiro Yokoyama, ${ }^{1}$ Ryoichi Hamasuna, ${ }^{1}$ Hajime Ohta, ${ }^{2}$ and Hideo Takeshima ${ }^{2}$

Objective: To retrospectively analyze the complication rates and risk factors associated with the use of the Angio-Seal vascular closure device during neuroendovascular therapy.

Methods: In this study, we enrolled 283 patients who underwent Angio-Seal hemostasis between December 2005 and June 2019 at our institute. We retrospectively analyzed the major and minor complication rates and risk factors between the complication and no-complication groups using the medical charts of patients for whom the device was used.

Results: Of the 283 patients, 5 had major complications (1.8\%) and 18 had minor complications (6.3\%). There were no significant differences between the complication $(n=23)$ and no-complication $(n=260)$ groups regarding the baseline characteristics or operation procedures. Among the major complications, superficial femoral artery puncture, $8 \mathrm{Fr}$ device, Angio-Seal Evolution, post-carotid artery stenting, dual antiplatelet therapy, and delirium were considered risk factors. Conclusion: The Angio-Seal is a safe and useful hemostatic device. However, puncture site complications need to be considered when the device is used for contraindicated patients or for those with delirium who cannot rest following the procedure.

Keywords > Angio-Seal, vascular closure device, complication

\section{Introduction}

Recently, the number of patients indicated for endovascular treatment has increased along with the development of devices for neuroendovascular treatment. As a measure to prevent perioperative thrombotic complications, oral antiplatelet administration or intraoperative heparin administration has become a requirement. In addition, with the increase in patients receiving intracranial thrombectomy

${ }^{1}$ Department of Neurosurgery, Saito-Koyu Medical Center, Saito, Miyazaki, Japan

${ }^{2}$ Department of Neurosurgery, Division of Clinical Neuroscience, Faculty of Medicine, University of Miyazaki, Miyazaki, Miyazaki, Japan

Received: July 12, 2019; Accepted: November 24, 2019 Corresponding author: Takahiro Yokoyama. Department of Neurosurgery, Saito-Koyu Medical Center, 1550 Tsuma, Saito, Miyazaki 881-0033, Japan

Email: takahiro_yokoyama@med.miyazaki-u.ac.jp

This work is licensed under a Creative Commons Attribution-NonCommercialNoDerivatives International License.

(C)2020 The Japanese Society for Neuroendovascular Therapy after intravenous recombinant tissue plasminogen activator (rt-PA) therapy for acute cerebral embolism, the frequency of the use of $8 \mathrm{Fr}$ or larger sheaths has increased, raising concerns over the increase in puncture site complications such as difficult hemostasis. Hemostatic devices and materials have been developed and distributed by many companies for speedy and consistent hemostasis at the puncture site, and based on their clinical application, their usefulness and safety have been reported. At our institution, we use Angio-Seal STS and Angio-Seal Evolution (both by Terumo Corporation), and have experienced puncture site complications. We, therefore, retrospectively evaluated the results of the use of the hemostatic devices at our institution, details of patients who had puncture site complications, and their risk factors with a review of the literature.

\section{Subjects and Methods}

Of the 285 patients who received neuroendovascular treatment at our hospital during the period from December 2005 to the end of June 2019 using Angio-Seal STS/Evolution for hemostasis, 283 were selected as the subjects after 
excluding those for whom the data of the evaluation items were missing. They were divided into those who developed puncture site complications (complication group) and those who did not (no-complication group). According to the classification method by Warren et al., ${ }^{1)}$ occlusion/ischemia, pseudoaneurysms, and arteriovenous fistulas that required changing hospital and surgical treatment or transfusion were defined as major complications, and bleeding/hematoma and infections that were able to be completely treated at our hospital were defined as minor complications. Regarding risk factors to be evaluated, the following information was obtained from the charts in our archives: (1) Patient background information including age, sex, body mass index (BMI), history of internal disorders, drinking and smoking history, and history of the use of antiplatelets/ anticoagulants and intravenous rt-PA, (2) surgical information such as the side of the puncture site, disease name, content of the procedure, operation time (time from entering to leaving the operation room), blood pressure upon leaving the operation room, and the presence of postoperative agitation. Concerning the size of the device used, a $6 \mathrm{Fr}$ Angio-Seal was selected when a $5 \mathrm{Fr}$ or $6 \mathrm{Fr}$ sheath was used, and an $8 \mathrm{~F}$ Angio-Seal was selected when a $7 \mathrm{Fr}$ or $8 \mathrm{Fr}$ sheath was used. Treatment using a 9Fr or larger sheath is not performed at our hospital.

Patient background information, surgical information, and the size of the Angio-Seal device used were compared between the complication and no-complication groups.

Before conducting this study, documents to be announced were put on the bulletin board in the hospital and on the hospital website, and approval by the ethical review board of the hospital (Approval No. Sairin 005) was received.

Statistical analysis was performed using Statistical Software $\mathrm{R}$. The $\mathrm{t}$-test was performed concerning the mean age, mean BMI, operation time, and postoperative systolic and diastolic blood pressures, and the chi-square test was performed concerning the other items. $p<0.05$ was defined as significant.

\section{Results}

Complications developed in $23(8.1 \%)$ of the 283 patients. The details of the complications are shown in Table 1. Five major complications were observed $(1.8 \%)$, consisting of four cases $(1.4 \%)$ of pseudoaneurysm formation and one case of femoral artery occlusion $(0.4 \%)$, and all required vascular surgery (Fig. 1). In all, 18 minor complications were observed (6.4\%), consisting of 16 cases $(5.7 \%)$ of puncture site bleeding/subcutaneous hematoma formation, 1 case $(0.4 \%)$ of puncture site infection, and 1 case $(0.4 \%)$ of device breakage (the string connecting the anchor and collagen broke, and the anchor migrated into a peripheral vessel of the lower extremity). Bleeding was observed at the puncture site from immediately after puncture in four, and bleeding or subcutaneous hematoma was noted at the puncture site after the patient returned to the hospital room after surgery in 12. Additional manual compression hemostasis was performed on these patients. For puncture site infection, antibiotics were administered. All minor complications were cured without developing into adverse events. The patient who had anchor migration was followed up, but no adverse events were observed.

Of the five major complications, four were associated with the use of the 8Fr Angio-Seal and were all pseudoaneurysm formation. In addition, three of the four pseudoaneurysm patients were administered two or more antiplatelet agents, and the remaining one developed the pseudoaneurysm after intravenous rt-PA therapy. None of them had a history of smoking, and four had a history of drinking. Three of the five patients underwent contraindicated superficial femoral artery puncture.

The background of the 23 patients in the complication group and 260 patients in the no-complication group is shown in Table 2. In the complication group, the mean age was 72.4 years, the proportion of men was $52.2 \%$, and the mean BMI was 23.3. In the no-complication group, they were 71.8 years, $55.8 \%$, and 23.8, respectively, and no significant difference was observed between the two groups. There was also no significant difference between the two groups in the history of hypertension, diabetes, dyslipidemia, hyperuricemia, or ischemic heart disease, underlying diseases, such as lower extremity peripheral artery disease, or their life history, including smoking and drinking habits, medications during treatment including antiplatelets and anticoagulants, and the number of patients given intravenous rt-PA therapy. Although the differences were not significant, the percentages of diabetic patients and those who used two or more antiplatelet agents were slightly higher in the complication group, and the percentage of patients with a history of dyslipidemia was slightly higher in the no-complication group.

Factors related to the surgical procedure between the two groups are compared in Table 3. No significant difference was observed in the side of puncture site, number of times of the use of Angio-Seal in the same vessel, size of the inserted sheath, size of Angio-Seal used, content of the 
surgical procedure, time from entering to leaving the operation room, or the blood pressure at leaving the operation room between the two groups. Although the percentage of patients treated using Angio-Seal Evolution was slightly higher in the complication group, the difference was not significant.

\section{Discussion}

If hemostasis is attempted at the puncture site by manual compression in patients perioperatively administered antithrombotic drugs, such as antiplatelets and anticoagulants, for endovascular surgery, time is needed until hemostasis is achieved due to hemorrhagic tendency, and the burden on the surgeon and assistants is increased. Hemostatic devices make quick and consistent hemostasis possible, and benefit both the patients and hospital staff.

The puncture site hemostatic devices manufactured and sold in Japan are classified into two types according to the hemostatic method and mechanism: (1) products to stop bleeding by occluding the external wall of the vessel at the puncture site using a bioabsorbable material (e.g., collagen, polyglycolic aid), and (2) products for direct suturing of the vascular wall at the puncture site using a non-absorbable suture. ${ }^{2)}$ Absorbable local hemostatic agents include AngioSeal and Exoseal (Cardinal Health Japan, Tokyo), and variants of Angio-Seal are Angio-Seal STS and Angio-Seal Evolution. As a non-absorbable suture set, Perclose ProGlide (Abott, Santa Clara, CA, USA) is available. Angio-Seal consists of an anchor and collagen sponge, which are connected by a suture, to stop bleeding by sandwiching the vascular wall at the puncture site with the anchor from inside and collagen sponge from the tissue side. ${ }^{3}$ Exoseal places a bioabsorbable polyglycolic acid plug for hemostasis outside the vessel and characteristically leaves no foreign material in the vessel. ${ }^{4}$ Perclose ProGlide applies a suture on both ends of the vascular wall at the puncture site and achieves hemostasis by advancing the knot to the vascular wall upon withdrawal of the device.5)

The results of the use of these hemostatic devices in coronary artery endovascular treatment have been reported. Warren et al. ${ }^{1)}$ conducted a single-center retrospective study of coronary artery endovascular treatment with hemostasis using Angio-Seal, and reported a major complication rate of $1.2 \%$ and a minor complication rate of $9 \%$. In addition, there have been multiple meta-analyses regarding coronary artery endovascular treatment. ${ }^{6-9)}$ Nikolsky et al. reported that complications were less frequent in the
Angio-Seal group than in the manual compression group, although the difference was not significant, and that the time until hemostasis, time of rest, and duration of hospitalization were shortened with a high degree of patient satisfaction in the Angio-Seal group. Das et al. ${ }^{7)}$ also reported that complications were less frequent in the Angio-seal group than in the manual compression group, although the difference was not significant, and that there were fewer ischemic complications of the lower extremities and complications that required surgical treatment. When the manual compression group and Perclose group were compared, the total complication rate was lower in the manual compression group, but the difference was not significant. However, the total complication rate did not differ significantly between the Angio-Seal group and Perclose group.

Concerning the results of the use of hemostatic materials after neuroendovascular treatment, Geyik et al. ${ }^{10)}$ reported that the major complication rate was $1.4 \%$ and the minor complication rate was $2.4 \%$ in the Angio-Seal group, in which 698 Angio-Seals were used. However, according to a multi-center study by Sato et al., ${ }^{11)}$ no major complications due to the use of Angio-Seal were observed after neuroendovascular treatment, and minor complications were observed in 3.4\% of the patients in the Angio-Seal group and in $1.8 \%$ of the patients in the manual compression group, with no significant difference between them. Furthermore, the time until hemostasis, time of bed rest, and time until discharge were shorter in the Angio-Seal group.

Thus, although the usefulness and safety of Angio-Seal have been established, puncture site complications have also been demonstrated to occur at a certain frequency. For example, the package insert mentions a vascular diameter of less than $4 \mathrm{~mm}$, puncture of the posterior wall, puncture of the superficial femoral artery, puncture at a point proximal to the inguinal ligament, marked calcification, and clotting abnormality as contraindications for Angio-Seal. As the inappropriate use of Angio-Seal leads to complications, proper selection of patients and careful application are required for its use. In this study, three of the five patients who developed major complications were found to have been punctured in the superficial femoral artery, which is a contraindication mentioned in the package insert, on close examination by CT-angiogram (CTA) after the development of complications, although this was not known during surgery.

However, puncture site complications have also been reported to occur at a certain frequency even when AngioSeal is used correctly. Concerning risk factors for complications, Warren et al. ${ }^{1)}$ reported that the frequency of 
Table 1 Summary of complications

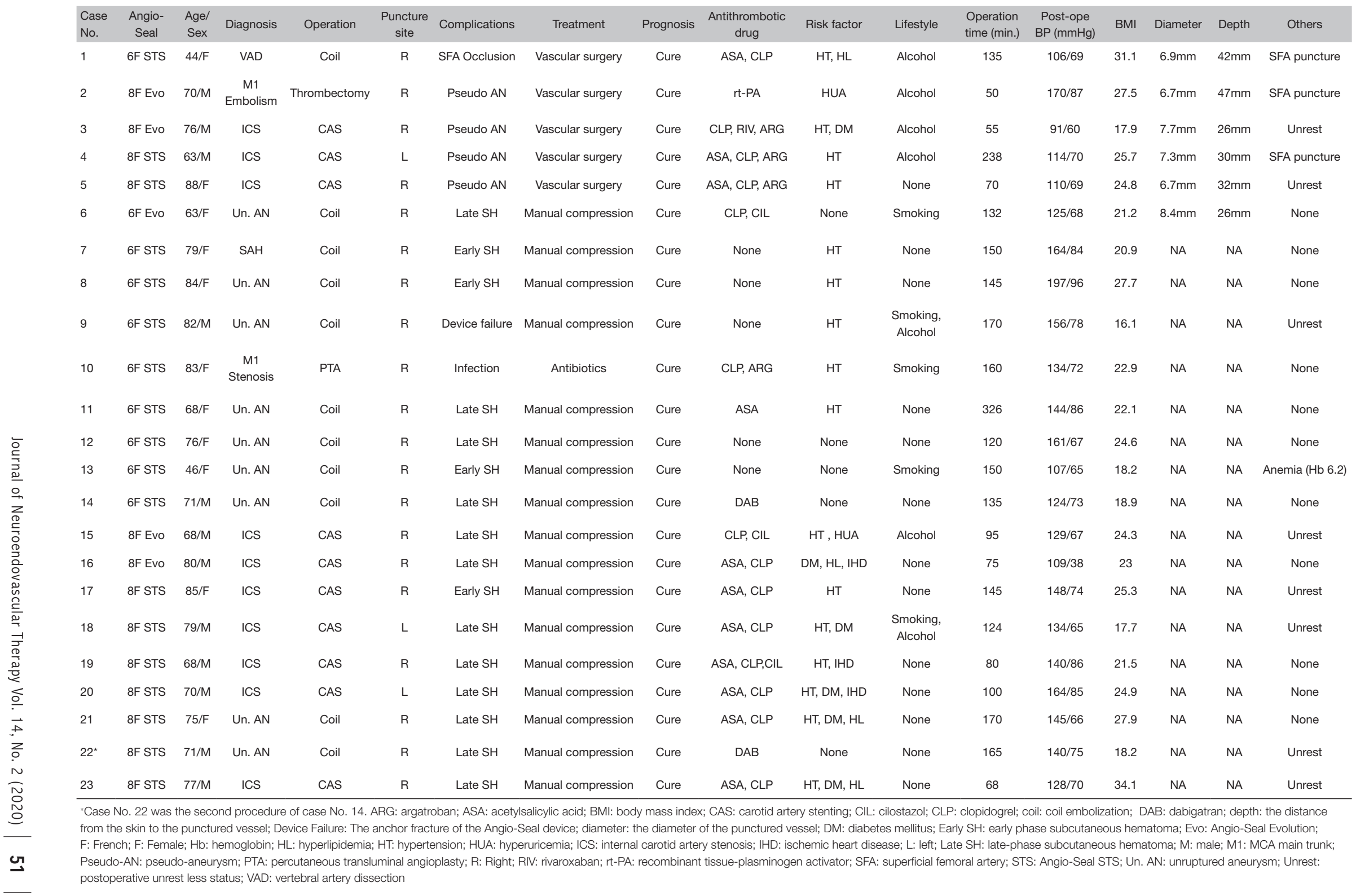



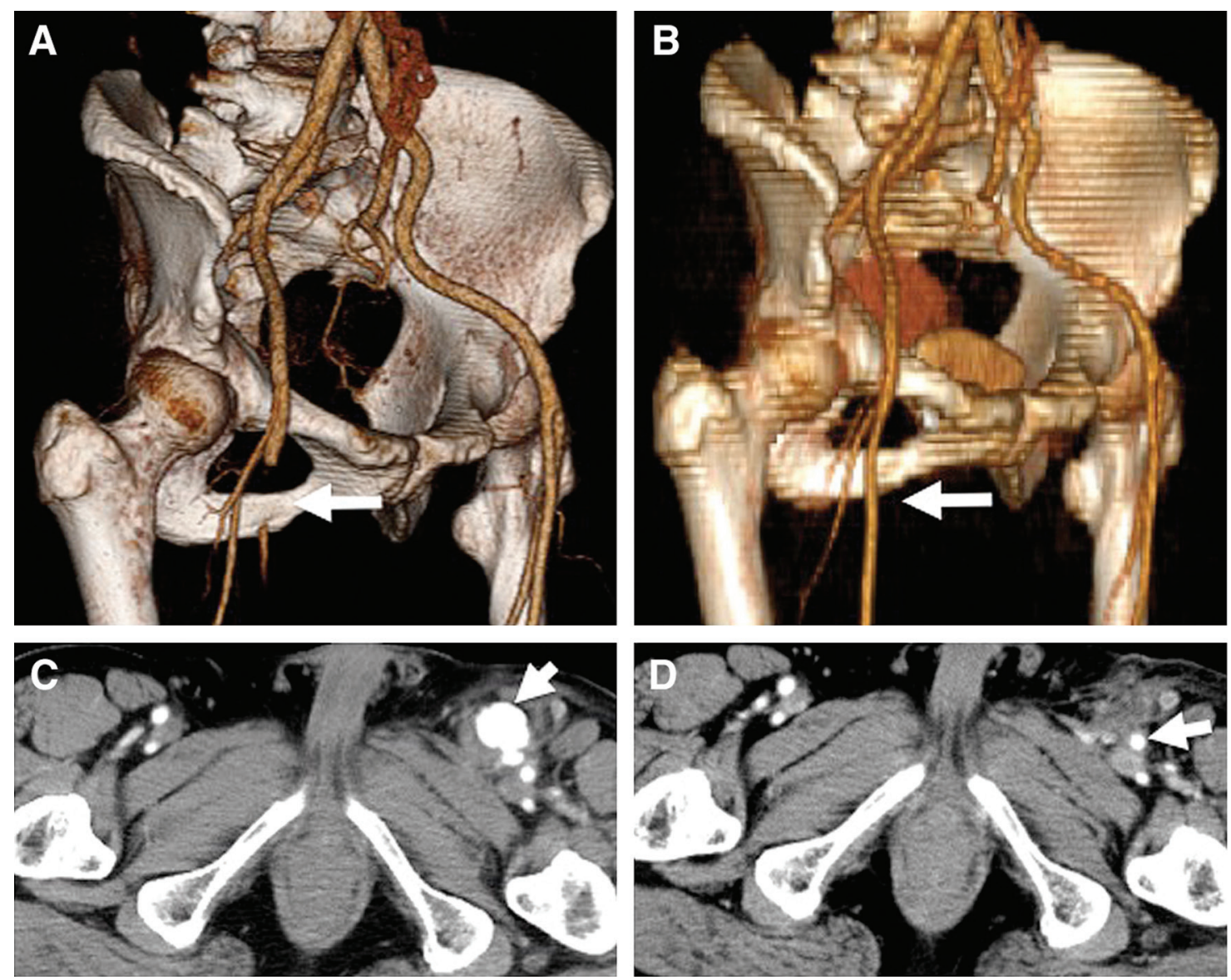

Fig. 1 Representative cases. (A) Case No. 1. Pelvic CT-angiogram before vascular surgery showed right SFA occlusion (white arrow) after the administration of the Angio-Seal STS hemostatic device. (B) Postoperative pelvic CT-angiogram showed the complete recanalization (white arrow) of the right SFA. (C) Case No. 4. Pelvic CT-angiogram before vascular surgery showed the pseudoaneurysm formation in the left SFA (white arrow) after the administration of the AngioSeal Evolution hemostatic device. (D) Postoperative pelvic CT-angiogram showed the complete repair of the left SFA (white arrow). CT: computed tomography; SFA: superficial femoral artery

complications was high in patients treated using an $8 \mathrm{Fr}$ sheath and in patients with a BMI of 28 or less. In addition, they reported that complications were more frequent in the first 50 patients treated using a device, suggesting the presence of a learning curve. Geyik et al. ${ }^{10)}$ reported that the major complication rates after the use of a diagnostic angiography and endovascular treatment were $0.13 \%$ and $1.4 \%$, respectively, and increased to $5.3 \%$ after treatment for cerebral aneurysms, and that one reason for the increase in complications was the perioperative use of antiplatelets and heparin. Sato et al. ${ }^{11)}$ also reported that the concomitant use of multiple antiplatelet drugs and large puncture sheath size can be risk factors. Moreover, Aida et al. ${ }^{12}$ found that a BMI of 21 or less and a distance to the femoral artery of less than $11.1 \mathrm{~mm}$ were risk factors for complications. The high frequency of complications in patients with a low BMI and those with a short distance from the skin to the femoral artery has been ascribed to insufficient hemostasis due to instability of the collagen sponge placed in lean patients with less subcutaneous tissue. The package insert of Angio-Seal ${ }^{3)}$ calls for caution in its use in not only lean but also obese patients. When using Angio-Seal STS, the collagen sponge is advanced with the tamper tube, and exposure of the tamping marker is confirmed. The tamper tube is $8.9 \mathrm{~cm}$ long, and the length from the tamping marker to the upper end of the collagen is approximately $8.2 \mathrm{~cm}$. The possibility of insufficient hemostasis has been suggested to increase when the length from below the skin to the vascular wall exceeds $7 \mathrm{~cm}$; therefore, caution is needed when using the device in obese patients.

According to the review of puncture site complications at our hospital, no significant risk factor was found related to the patient background or surgical procedure. However, complications developed more frequently in diabetic patients, patients administered multiple antiplatelet drugs, those using an $8 \mathrm{Fr}$ device, those treated using Angio-Seal 
Table 2 Comparison of patient background between the complication group and the no complication group

\begin{tabular}{|c|c|c|c|}
\hline & Complication group $(n=23)$ & No-complication group $(n=260)$ & $P$ value \\
\hline Mean age (y.o.) & 72.4 & 71.8 & $p=0.79$ \\
\hline Male (\%) & $12(52.2 \%)$ & $145(55.8 \%)$ & $p=0.91$ \\
\hline Hypertension & $16(70.0 \%)$ & $184(70.8 \%)$ & $p=1$ \\
\hline Diabetic mielitis & $6(26.1 \%)$ & $40(15.4 \%)$ & $p=0.29$ \\
\hline Hyperlipidemia & $4(17.4 \%)$ & $79(30.4 \%)$ & $p=0.28$ \\
\hline Hyperulemia & $2(8.7 \%)$ & $39(15 \%)$ & $p=0.6$ \\
\hline Coronary artery disease & $3(13.4 \%)$ & $38(14.2 \%)$ & $p=1$ \\
\hline Peripheral artery disease & $0(0 \%)$ & $10(3.8 \%)$ & $p=0.71$ \\
\hline Smoking & $5(21.7 \%)$ & $52(20 \%)$ & $p=1$ \\
\hline Alcohol & $6(26.1 \%)$ & $109(41.9 \%)$ & $p=0.21$ \\
\hline Single antiplatelet drug & $1(4.3 \%)$ & $44(16.9 \%)$ & $p=0.19$ \\
\hline Antiplatelet drug + Anticoagulant drugs & $1(4.3 \%)$ & $14(5.4 \%)$ & $p=1$ \\
\hline Single anticoagulant drug & $2(8.7 \%)$ & $13(5.0 \%)$ & $p=0.78$ \\
\hline i.v. rt-PA & $1(4.3 \%)$ & $26(10 \%)$ & $p=0.6$ \\
\hline
\end{tabular}

There are no significant statistical differences between two groups. BMl: body mass index; i.v. rt-PA: intravenous recombinant tissue Plasminogen

activator injection; y.o.: years-old

Table 3 Comparison of surgical factors between the complication group and the no complication group

\begin{tabular}{|c|c|c|c|}
\hline & Complication group $(n=23)$ & No-complication group $(n=260)$ & $P$ value \\
\hline \multicolumn{4}{|l|}{ Femoral puncture site } \\
\hline Right (\%) & $20(86.9 \%)$ & $243(93.5 \%)$ & $P=1$ \\
\hline Multiple session operation & $1(4.3 \%)$ & $15(5.8 \%)$ & $P=1$ \\
\hline \multicolumn{4}{|l|}{ Sheath size } \\
\hline $5 \mathrm{~F}$ & $1(4.3 \%)$ & $7(2.7 \%)$ & $P=1$ \\
\hline $6 \mathrm{~F}$ & $9(39.1 \%)$ & $118(45.4 \%)$ & $P=0.71$ \\
\hline $7 \mathrm{~F}$ & $1(4.3 \%)$ & $6(2.3 \%)$ & $P=1$ \\
\hline $8 \mathrm{~F}$ & $12(52.2 \%)$ & $129(49.6 \%)$ & $P=0.98$ \\
\hline \multicolumn{4}{|l|}{ Angio-Seal Size } \\
\hline $6 \mathrm{~F}$ & $10(43.5 \%)$ & $125(48.1 \%)$ & $P=0.83$ \\
\hline $8 \mathrm{~F}$ & $13(56.5 \%)$ & 135 (51.9\%) & $P=0.83$ \\
\hline Use of Angio-Seal Evolution (\%) & $5(21.7 \%)$ & $25(9.6 \%)$ & $P=0.14$ \\
\hline Operation Time (minutes) & 127 & 135.1 & $P=0.52$ \\
\hline Mean postoperative Systolic BP (mmHg) & 136.5 & 134.9 & $P=0.75$ \\
\hline Mean postoperative diastolic BP $(\mathrm{mmHg})$ & 72.6 & 73.7 & $P=0.71$ \\
\hline \multicolumn{4}{|l|}{ Endovascular surgery } \\
\hline Coil embolization & $11(47.8 \%)$ & $123(47.3 \%)$ & $P=1$ \\
\hline CAS & $10(43.5 \%)$ & $82(31.5 \%)$ & $P=0.34$ \\
\hline Thrombectomy & $1(4.3 \%)$ & $46(17.7 \%)$ & $P=0.17$ \\
\hline Others & $1(4.3 \%)$ & $9(3.5 \%)$ & $P=1$ \\
\hline Within 50 cases from initial device usage & $7(16.2 \%)$ & $43(16.5 \%)$ & $P=0.6$ \\
\hline
\end{tabular}

There are no significant statistical differences between two groups. F: French; Operation time: the time from entry to exit in Angio suite; CAS: carotid artery stenting; Thrombectomy: mechanical thrombectomy with the stent-retriever

Evolution, and those who underwent CAS, as shown in Tables 2 and 3. As reported previously, we consider it necessary to perform hemostatic treatment carefully with attention to the occurrence of complications when AngioSeal is used concomitantly with a device with a large sheath diameter in patients administered multiple antiplatelet drugs. The effects of other factors, including the surgical procedure, operation time, and blood pressure, were suggested to be small. In addition, as no increase in puncture site complications associated with intravenous rt-PA therapy was observed, Angio-Seal is also considered to be relatively safe for patients administered rt-PA. 
In the five patients in the Angio-Seal group who developed major complications, the mean BMI was 25.4 and the mean distance from the skin to the vascular wall was $35.4 \mathrm{~mm}$, which were not consistent with previously reported risk factors related to the body type or anatomical structure of the puncture site such as the BMI, vascular lumen, and depth from the skin. However, three patients were found to have undergone superficial femoral artery puncture, which is a contraindication, on close evaluation by CTA after the development of complications. In retrospect, we should have performed surgery after excluding superficial femoral artery puncture by sheath imaging after its placement to be compliant with the indications. As we presently perform sheath imaging for all patients, this complication is expected to become less common in the future. However, no contraindication or risk factor that may have caused complications was found in the remaining two patients, and another possible factor is that the patients were agitated immediately after the use of Angio-Seal and were unable to be sedated. Postoperative agitation was observed in 8 (34.7\%) of the 23 patients who developed complications, which is considered a possible risk factor for complications. The inability to rest the puncture site due to agitation may cause detachment of collagen from the puncture site before hemostasis, leading to puncture site bleeding or pseudoaneurysm formation.

At our hospital, preoperative imaging examination of the puncture site was not performed. The incidence of major complications is not high and the necessity of uniform preoperative evaluation is considered low, but evaluation of the puncture site using ultrasound, which is mildly invasive and easy to perform, or ultrasound-guided puncture may be useful. Furthermore, it is considered necessary to assess contraindications by examining the puncture site by fluoroscopy and performing sheath imaging after puncture. If the superficial femoral artery is found to have been punctured by this examination, it is necessary to re-puncture at another site. In addition, in high-risk patients with advanced atherosclerosis, it is also necessary to consider surgical exposure of the femoral artery and direct suturing of the puncture site. Patients who have undergone clot retrieval and elderly patients with cognitive impairment often become agitated after surgery. For restless patients who may accidentally flex the puncture site, sufficient sedation until hemostasis is achieved should be considered.

Although Angio-Seal is a safe hemostatic device, it is necessary to accumulate experience of its use, correctly perform preoperative examinations, comply with its indications, and evaluate reliable hemostatic methods to further reduce the complication rate.

\section{Conclusion}

Angio-Seal is a hemostatic device that can be used safely with sufficient knowledge about its structure and method of use. With Angio-Seal, hemostasis can be achieved quickly even in patients in whom hemostasis is considered difficult such as those undergoing intravenous rt-PA therapy. However, caution of the increased possibility of puncture site complications is needed for patients who have received contraindicated treatments, such as superficial femoral artery puncture, and those who are restless due to postoperative agitation. For the safer use of Angio-Seal, careful puncture and compliance with indications are necessary.

\section{Disclosure Statement}

The first author and all coauthors have no conflicts of interest.

\section{References}

1) Warren BS, Warren SG, Miller SD: Predictors of complications and learning curve using the Angio-Seal closure device following interventional and diagnostic catheterization. Catheter Cardiovasc Interv 1999; 48: 162-166.

2) Pharmaceuticals and Medical Devices Agency, Precautions for Use of Puncture Site Closure Device, Pharmaceuticals and Medical Devices Safety Information, 2014;309:3-6.

3) Terumo Corporation, Angio-Seal ${ }^{\mathrm{TM}}$ package insert (revised on June, 2017), 2017. https://www.info.pmda.go.jp/ downfiles/md/PDF/470034/470034_21900BZY00056000 A_09_02.pdf.

4) Cordis ExosealTM package insert. 2017. https://www. cordisjapan.jp/content/dam/cordis/web/documents/ifu/ cordis-jp-exoseal-ifu.pdf.

5) Japan AV. PROGLIDE package insert, 2016. https://www. info.pmda.go.jp/downfiles/md/PDF/340733/340733_ 21900BZY00065000_A_01_05.pdf.

6) Nikolsky E, Mehran R, Halkin A, et al: Vascular complications associated with arteriotomy closure devices in patients undergoing percutaneous coronary procedures: a meta-analysis. J Am Coll Cardiol 2004; 44: 1200-1209.

7) Das R, Ahmed K, Athanasiou $T$, et al: Arterial closure devices versus manual compression for femoral haemostasis in interventional radiological procedures: a systematic review and meta-analysis. Cardiovasc Intervent Radiol 2011; 34: 723-738. 
8) Vaitkus PT: A meta-analysis of percutaneous vascular closure devices after diagnostic catheterization and percutaneous coronary intervention. $J$ Invasive Cardiol 2004; 16: 243-246.

9) Koreny M, Riedmüller E, Nikfardjam M, et al: Arterial puncture closing devices compared with standard manual compression after cardiac catheterization: systematic review and meta-analysis. JAMA 2004; 291: 350-357.

10) Geyik S, Yavuz K, Akgoz A, et al: The safety and efficacy of the Angio-Seal closure device in diagnostic and interventional neuroangiography setting: a single-center experience with 1,443 closures. Neuroradiology 2007; 49: 739-746.

11) Sato M, Matsumaru $Y$, Sakai N, et al: Usefulness of an access-site hemostasis device in neuroendovascular treatment. Acta Neurochir (Wien) 2017; 159: 2331-2335.

12) Aida $Y$, Misaki K, Kamide T, et al: Physical risk factors of hemorrhagic complications associated with angio-seal closure device use in neurointerventional procedures. World Neurosurg 2018; 111: e850-e855. 